\title{
Experimental studies of the effects of extrinsic factors on conduction in normal and demyelinated nerve
}

\author{
1. Temperature \\ F. A. DAVIS, C. L. SCHAUF, B. J. REED, AND R. L. KESLER \\ From the Departments of Neurological Sciences and Physiology, Rush Medical College, \\ Chicago, Illinois, 60612, USA
}

SYNOPSIS Previous studies in experimentally demyelinated mammalian nerves have demonstrated that a reversible conduction block occurs with small increases of temperature within the animal's normal body temperature range. This phenomenon is believed to be the mechanism for clinical temperature effects in multiple sclerosis. This study examines some quantitative thermal relationships in demyelinated nerves of guinea pigs with experimental allergic neuritis. The observed results in normal and experimental animals are in good agreement with previous theoretical calculations based on the effects of temperature on the voltage and time-dependent behaviour of the ionic permeabilities of the nodes of Ranvier. Guinea pigs with increasing motor dysfunction generally exhibited corresponding increases in the overall latency of the conducted action potential, as well as decreases in amplitude. In addition, the lower the initial velocity increment per degree of temperature elevation, the lower was the temperature at which conduction block began to occur. Except for a few cases in which the recorded action potential was bimodal, with response at both normal and prolonged latency, the results tended to indicate a remarkedly uniform involvement of the sciatic nerve within the region of temperature control.

In a previous communication (Schauf and Davis, 1974) we demonstrated that a numerical solution of the Frankenhauser-Huxley equations for conduction in vertebrate myelinated nerve fibres (Frankenhauser and Huxley, 1964; Goldman and Albus, 1968) modified solely by alterations in passive cable properties (Smith and Koles, 1970; Koles and Rasminsky, 1972), predicted a complex relationship between the extent of demyelination and the temperature dependence of conduction in this model system. In particular, Fig. 1 of Schauf and Davis (1974) predicts that with increasing loss of myelin there should occur decreases in (1) conduction velocity at a given temperature, (2) slope of the conduction velocity-temperature relation, and (3) blocking temperature.

Experimental verification of these predictions is limited and not systematic. Thus Davis and
Jacobson (1971) first demonstrated in guinea pigs with experimental allergic neuritis (EAN) that conduction block could occur at a temperature $10-15^{\circ} \mathrm{C}$ lower than in normal animals, but did not attempt to correlate blocking temperature with the extent of demyelination. Later, Rasminsky (1973), using diphtheria toxin to induce demyelination, observed that fibres with long internodal conduction times could be blocked by increases in temperature which in less severely affected fibres caused an increase in conduction velocity.

To provide additional experimental justification for the use of such a theoretical model in predicting the effects of extrinsic factors on conduction in demyelinated nerve, we have undertaken a more detailed series of studies of the effect of temperature on conduction in the sciatic nerve of guinea pigs with EAN. The results not only provide support for the model 
system, but also for the usefulness of the EAN multifibre preparation in studying quantitative aspects of conduction in demyelinated nerve.

\section{METHODS}

Experimental allergic neuritis was induced in 400 $500 \mathrm{~g}$ adult male guinea pigs by the method of Waksman and Adams (1956). Using aseptic techniques $1 \mathrm{~g}$ of rabbit sciatic nerve was cut into short lengths, then further sliced in a freezing microtome set at $5 \mu \mathrm{m}$ advancements. To this tissue was added $2.5 \mathrm{ml}$ isotonic saline and $5 \mathrm{ml}$ complete Freund's adjuvant (Difco). The mixture was emulsified for five minutes in a cold electric blender. Each guinea pig was injected intradermally with $0.1-0.15 \mathrm{ml}$ of the emulsion in each forefoot pad within 30 minutes after preparation. Overall, $35 \%$ of the 47 injected animals developed severe paresis, principally in the hind limbs, to the extent that motor function was almost entirely absent. Milder reactions, causing difficulty in the ability to right themselves and ambulate was noted in $30 \%$ of the animals, while $20 \%$ exhibited no overt signs of nerve dysfunction. Approximately $15 \%$ of the injected animals died before use. There was no apparent difference in the results when using fresh rabbit sciatic nerve or a commercially supplied frozen product (Pel-Freeze).

Recording techniques were a modification of previous methods (Davis and Jacobson, 1971). Animals were given sodium pentobarbitone anaesthesia intraperitoneally and the sciatic nerve and its peroneal branch exposed by two separate incisions in the thigh and tibial regions respectively. A tracheostomy was performed. The sciatic nerve was severed near its exit from the cord at the apex of the thigh and a pair of platinum stimulating electrodes applied at this point. A mixture of Vaseline and mineral oil was used to insulate these electrodes from the remainder of the thigh wound, the latter being arranged to form a test pool 1-1.5 cm in length surrounding the more distal portion of the sciatic nerve. Heated Ringer solution was then constantly circulated through this pool at flow rates of $10-20 \mathrm{ml} / \mathrm{min}$ and removed by suction. Temperature was monitored by a thermistor placed in the test pool near the nerve trunk. An earth (ground) electrode was also inserted into this pool. Recording electrodes were placed along the peroneal branch of the sciatic, the more proximal electrode being located just distal to the bifurcation of the sciatic nerve into its peroneal and tibial branches. The other electrode was placed on a crushed segment of the peroneal nerve approximately $1 \mathrm{~cm}$ distal to the active electrodes. Vaseline was again used as an insulator.
Animals were next given sufficient D-tubocurarine intraperitoneally to eliminate movement or muscle action potentials and placed on a rodent respirator (Harvard Apparatus). The distance between the stimulating and proximal recording electrode was recorded as was the overall length of nerve bathed by heated Ringer solution.

In a series of control experiments, the temperature of the test pool was raised to $45^{\circ} \mathrm{C}$ while additional thermistors monitored temperatures near the recording sites. Temperatures in these uncontrolled regions of the preparation remained at $25-27^{\circ} \mathrm{C}$ independently of changes produced in the test pool. Room temperature was $22-24^{\circ} \mathrm{C}$.

An estimate of conduction velocity as a function of test pool temperature can be obtained by measuring the latency of the conducted compound action potentials and employing the following argument. If $L$ is the total fibre length and $l$ is the length of nerve subject to alterations in temperature, then if one measures the latency $\left(\Delta t_{0}\right)$ with the entire preparation at a single temperature $\left(T=25-27^{\circ} \mathrm{C}\right)$ and the latency $(\Delta t)$ at any other temperature $(T)$, we can estimate the conduction velocity at $T_{0}$ as

$$
\theta_{0}=\frac{L}{t_{0}}
$$

Then, if we assume that no change in temperature occurs outside the test pool, the latency at higher temperature should be approximately:

$$
\Delta t=\frac{l}{\theta_{t}}+\frac{L-l}{\theta_{0}}
$$

where $\theta_{t}$ is the conduction velocity at temperature $T$ in nerve segment $l$, and solving for $\theta_{t}$ while using (1) gives

$$
\theta_{\iota}=\frac{l}{\Delta t-\Delta t_{0}(1-l / L)}
$$

as a function of measurable parameters.

This argument is obviously subject to some limitations. The length $L$ can be measured after an experiment with some accuracy, but $l$ is much less well defined, since it is unclear how far beyond the test pool the effects of temperature changes could extend. We tried to minimize this by appropriate use of vaseline seals. We have minimized the initial latency between stimulus onset and initiation of nerve activity by using supramaximal stimuli three to four times larger than required for a maximum response.

Note that, in the experiments using EAN animals, we always determined blocking temperature in addition to calculating the $\theta(T)$ relation since a blocking temperature above $45^{\circ} \mathrm{C}$ (where normal fibres begin to block), combined with a large latency at $25^{\circ} \mathrm{C}$ and a low slope of the $\theta(T)$ curve could be accounted for if we were recording from residual, normal small 
diameter fibres. This was only occasionally seen and the preparation was not used. Generally, responses of long latency were observed to block at temperatures below $40^{\circ} \mathrm{C}$, thus indicating a major contribution of demyelinated fibres to the recorded response.

\section{RESULTS}

Figures 1 and 2 show a series of records of the compound action potentials recorded at increasing temperatures in a normal animal and in an animal with experimental allergic neuritis. The normal response typically has an initial latency of about $0.8 \mathrm{~ms}$ at $25^{\circ} \mathrm{C}$, and is blocked by temperatures of $44-48^{\circ} \mathrm{C}$. The latency in EAN animals is, as expected, much longer, ranging from $1.5-4.0 \mathrm{~ms}$ at $25^{\circ} \mathrm{C}$. Conduction block in
EAN animals occurs at temperature as low as $34-37^{\circ} \mathrm{C}$. The table contains data on the severity of clinical signs, latency at $25^{\circ} \mathrm{C}$, and blocking temperature. Generally, there was a very good correlation between the overt clinical behaviour and the associated increase in latency and decrease in blocking temperature.

Perhaps the most interesting feature of Fig. 2 is the appearance of a fairly well-defined peak in the compound action potential, suggesting a surprisingly uniform dysfunction of the sciatic nerve in these animals averaged over the length of nerve fibre under investigation. That this response is not a response from non-demyelinated nerve fibres of smaller diameter is shown by the fact that the response is blocked at tempera-

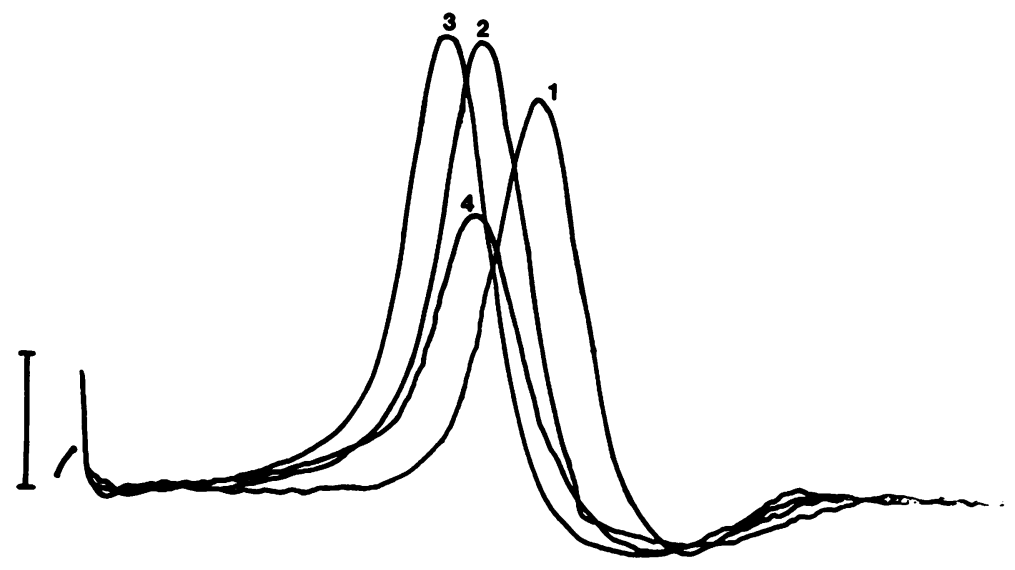

FIG. 1 Compound action potentials at increasing temperatures recorded from the peroneal nerve of a normal animal in response to supramaximal stimulation of the proximal end of the sciatic nerve. Horizontal and vertical calibrations were $0.2 \mathrm{~ms}$ and $0.5 \mathrm{mV}$ respectively. Records $1-4$ were taken at temperatures of $25,35,44$, and $48^{\circ} \mathrm{C}$.

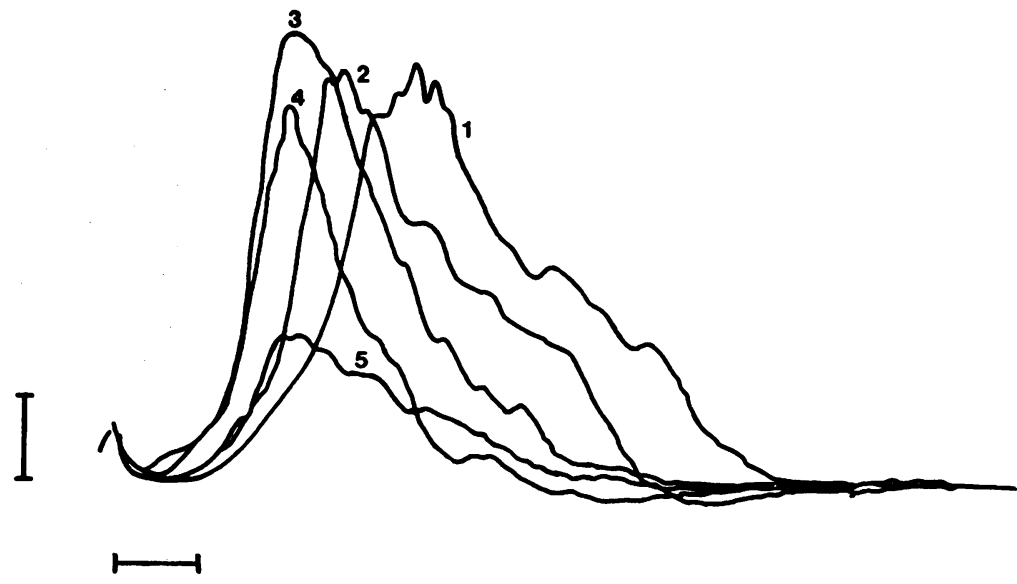

FIG. 2 Compound action potentials at increasing temperatures recorded from an animal with EAN. Horizontal and vertical calibrations were $1.0 \mathrm{~ms}$ and $0.05 \mathrm{mV}$ respectively. Records 1-5 were taken at temperatures of $20,26,35$, 39 , and $40^{\circ} \mathrm{C}$. 
TABLE

CLINICAL APPEARANCE, ACTION POTENTIAL LATENCY, AND BLOCKING TEMPERATURE IN GUINEA PIGS WITH EXPERIMENTAL ALLERGIC NEURITIS

\begin{tabular}{lccc}
\hline Animal & Condition & $\begin{array}{c}\text { Latency at } 25^{\circ} \mathrm{C} \\
(\mathrm{ms})\end{array}$ & $\begin{array}{c}\text { Blocking } \\
\text { temperature } \\
\left({ }^{\circ} \mathrm{C}\right)\end{array}$ \\
\hline 74GP5 & +++ & 4.2 & $35 \pm 2$ \\
74GP6 & +++ & 2.0 & $38 \pm 2$ \\
74GP7 & +++ & 4.8 & $35 \pm 2$ \\
74GP10 & +++ & 3.4 & $37 \pm 1$ \\
74GP3 & ++ & 3.8 & $40 \pm 1$ \\
74GP11 & ++ & 2.4 & $39 \pm 1$ \\
74GP14 & ++ & 1.8 & $39 \pm 1$ \\
74GP4 & + & 1.3 & $40 \pm 1$ \\
74GP8 & + & 1.5 & $39 \pm 1$ \\
75GP2 & + & 1.5 & $39 \pm 1$ \\
75GP3 & + & 1.3 & $40 \pm 1$ \\
74GP9 & $\mathrm{R}$ & 1.0 & $41 \pm 1$ \\
74GP12 & 0 & 1.0 & $45 \pm 1$ \\
74GP13 & 0 & 0.65 & $46 \pm 1$ \\
75GP1 & 0 & 0.75 & $43 \pm 1$ \\
75GP4 & 0 & 0.80 & $45 \pm 1$ \\
\hline
\end{tabular}

+++ Little or no motor function in hind limbs.

++ Poor gait; difficulty in righting; unable fully to support weight. + Mild gait impairment; only slight difficulty in righting. $R$ Injected. Little or no clinical sign.

0 Uninjected control.

tures $7-8^{\circ} \mathrm{C}$ cooler than those required to block such responses in normal animals.

In some animals, we did observe a multicomponent compound action potential with peaks both at near normal and increased latency, which is what one might expect if only a fraction of the sciatic nerve fibres were demyelinated. In this case, the components of long latency were blocked at substantially lower temperatures than the earlier response, confirming previous observations (Davis and Jacobson, 1971).

In Figs 1 and 2 the response latency decreases with increased temperature. Application of Equation 3 to these and similar data produces the result shown in Fig. 3. Here is plotted the calculated conduction velocity of the nerve fibre in the test pool as a function of test pool temperature for all animals examined. Each straight line represents data from a single preparation with symbols being altered to avoid confusion. The lowest set of data were obtained from animals lacking almost all motor function in their hind limbs $(+++$ in Table 1$)$, while the upper curves are from normal animals. The intermediate data were obtained from injected animals having varying gait impairment and some difficulty in righting $(++$ and + in Table

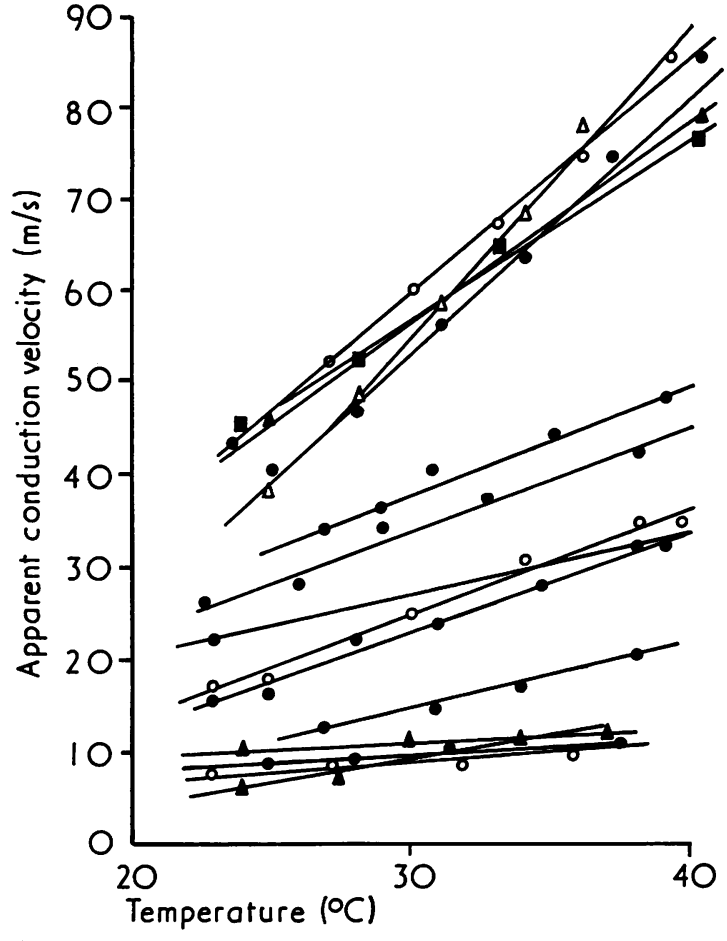

FIG. 3 The apparent conduction velocity as a function of temperature calculated using equation three from records similar to those in the previous two figures. Each line represents a different animal. Symbols have been changed where possible confusion exists. The straight lines are drawn by eye. Data are plotted only up to $40^{\circ} \mathrm{C}$ for animals with blocking temperatures greater than this.

1), but still with considerable motor function remaining.

The data compare well with the calculated responses of the model system of Schauf and Davis (1974). In both cases, the lower the conduction velocity at any particular temperaturefor example, $25^{\circ} \mathrm{C}$ - the smaller the rate of increase of conduction velocity with temperature. Lower conduction velocities at $25^{\circ} \mathrm{C}$ are theoretically a measure of increasing severity or extent of demyelination. In order to compare these data with the calculated theoretical behaviour, we have plotted in Fig. 4 (solid symbols) the slope of the conduction velocity-temperature curves of Fig. 3 as a function of the conduction velocity at $37^{\circ} \mathrm{C}$. The result is approximately 




FIG. 4 The solid symbols give the rate of increase of conduction velocity with temperature, calculated from the data of Fig. 3, as a function of conduction velocity at $37^{\circ} \mathrm{C}$. The open symbols have been obtained from the theoretical calculations of Schauf and Davis (1974) but at a reference temperature of $20^{\circ} \mathrm{C}$. The solid line is the least-squares fit to the solid symbols.

linear. Note that the slope of the line in Fig. 4 is critically dependent on the temperature at which the slopes of the velocity-temperature curves are measured and will vary accordingly.

One can obtain a similar plot from the theoretical calculations of Schauf and Davis (1971). In this case, a linear relationship is again obtained, suggesting a close correspondence between the model and real demyelinated fibre. Of course, here also the slope is dependent on the reference temperature chosen. If $37^{\circ} \mathrm{C}$ were selected as the reference temperature for the frog, the slope would be less than for the experimental data. However, the theoretical calculations rely on an empirical description of frog myelinated nerve at $20^{\circ} \mathrm{C}$. If this is taken as the reference temperature the results (open symbols in Fig. 4) agree very closely with the experimental findings. This superposition could, of course, be fortuitous, or perhaps it reflects some fundamental adjustment of the different species to their markedly dissimilar body temperatures. In any case, the important feature is the linear behaviour present in both cases.

In Fig. 5 is plotted the blocking temperatures as a function of conduction velocity at $25^{\circ} \mathrm{C}$, since the latter is presumably a measure of the

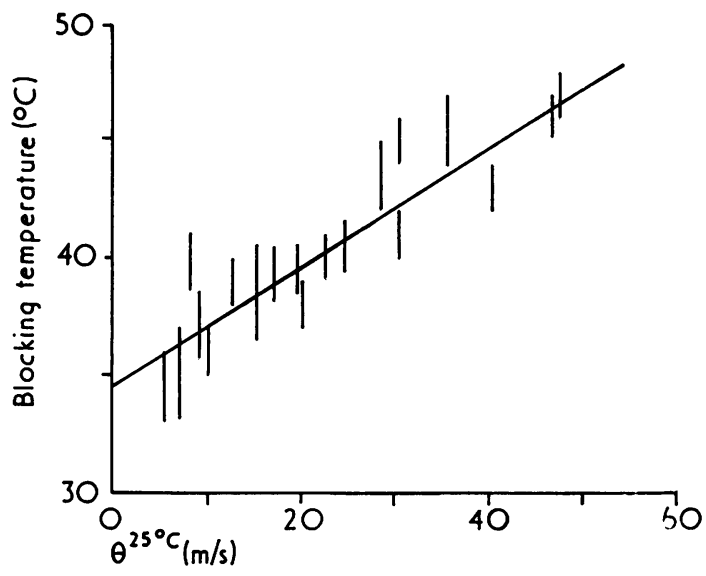

FIG. 5 Experimentally measured blocking temperatures as a function of conduction velocity at $25^{\circ} \mathrm{C}$. The vertical lines represent the uncertainty of measurement. The solid line is drawn by eye.

severity of demyelination. The vertical lines represent the uncertainty of measurement. Clearly, the blocking temperature increases as the conduction velocity increases, the experimental relationship being nearly linear. Figure 6 shows the theoretical relation between blocking temperature and conduction velocity calculated

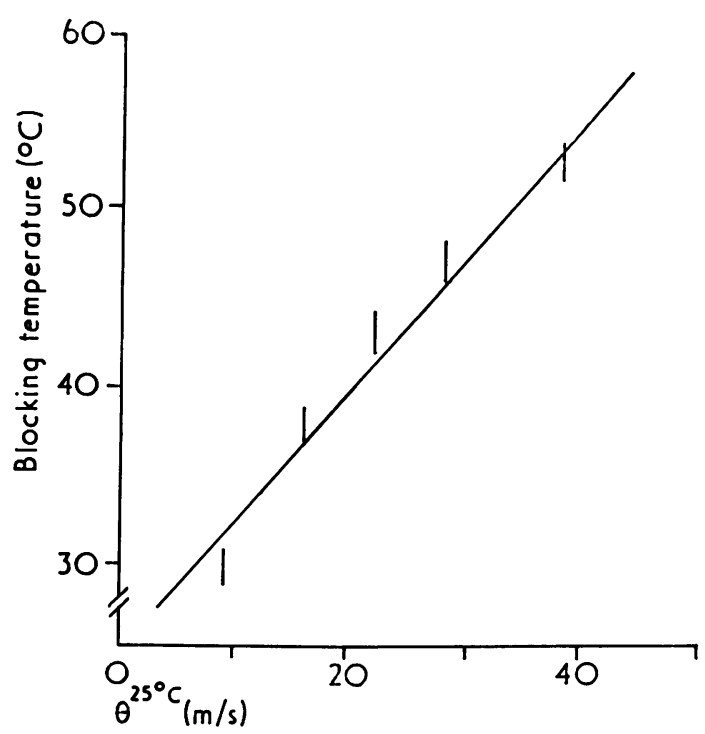

FIG. 6 Theoretical relationship between blocking temperature and conduction velocity at $25^{\circ} \mathrm{C}$ from the data of Schauf and Davis (1974). Compare with Fig. 5. 
from our model. There seems to be no great difference in the form of the relations. Both are nearly linear, again providing support for the continued use of the theoretical model in analysing conduction in demyelinated nerve.

\section{DISCUSSION}

A detailed understanding of impulse conduction abnormalities such as conduction slowing and block in demyelinated axons was provided by a systematic investigation of the behaviour of a standard mathematical model for nerve conduction modified for demyelinated fibres (Schauf and Davis, 1974). It was shown that the conduction characteristics of a demyelinated nerve are the expected consequences of myelin loss and the known ionic conductance kinetics of the excitable membrane. The present study provides quantitative data in support of this mathematical model.

We have shown that the model's prediction for the effects of temperature on conduction velocity and conduction block are precisely fulfilled. Specifically, it has been experimentally verified that the lower the conduction velocity in the demyelinated EAN preparation, the lower is the slope for conduction velocity increase with temperature rise and the greater is the depression of the temperature at which block occurs. Furthermore, as predicted by the model, these effects are linear functions.

An important aspect of this study is the demonstration that precise quantitative relationships for single fibre behaviour may be obtained from a multifibred experimental preparation. This would appear to be possible due to a similar degree of dysfunction in a majority of the demyelinated fibres. This is apparent from the single well-defined peak in the compound action potential in the majority of the EAN preparations. We intend to employ this method in further exploring the effects of other extrinsic factors on conduction in demyelinated nerve. Of particular interest will be the exploration of drugs and chemicals which are predicted by the experimental model to improve conduction in demyelinated nerve. These include agents with the ability to increase the membrane current either by slowing the sodium inactivation process or by inhibiting the potassium activation process (Schauf and Davis, 1974).

It is generally accepted that the clinical signs and symptoms in multiple sclerosis are largely the result of defects in impulse conduction in demyelinated CNS axons. A striking example of this is the central visual field defect associated with optic nerve lesions. Here there can be little doubt that the observed scotoma is related to a disturbance of conduction in a specific bundle of topographically oriented demyelinated fibres. With respect to the well-known clinical worsening with hyperthermia in multiple sclerosis the relationship of conduction velocity and conduction block to temperature suggests that the clinical phenomenon is due to a block of conduction and not to a change in conduction velocity. Since the low conduction velocity in demyelinated axons increases with temperature elevation it should, if anything, tend to improve clinical signs and symptoms. That the net effect of hyperthermia is a worsening is not surprising, in that the change in conduction velocity with a small change in body temperature will probably not exceed several metres per second, a minor effect compared with the block of conduction produced as the result of the decrease in action potential duration with increasing temperature. The seemingly paradoxical response of a nerve fibre to increasing temperature is largely due to the fact that the increased rate of rise of the initial phase of the action potential is a more important determinant of the internodal conduction time than is the amplitude or duration of the action potential. This is not to say that slowing of conduction is without importance in clinical function in the normothermic patient with multiple sclerosis. Indeed, the Pulfrich phenomenon in optic neuritis is probably best understood in terms of slowing of conduction velocity in the affected optic nerve fibres (Frisen et al., 1973). In this phenomenon, a pendulum bob swinging in a frontal plane viewed binocularly seems to move along an elliptical orbit away from the involved eye.

These studies were supported by the Morris Multiple Sclerosis Research Fund and by a grant (RG 921-A-1) from the National Multiple Sclerosis Society. C. L. S. is the recipient of a Research Career Development Award from the National Institutes of Health (1 K04 NS0000401). 


\section{REFERENCES}

Davis, F. A., and Jacobson, S. (1971). Altered thermal sensitivity in injured and demyelinated nerve. Journal of Neurology, Neurosurgery, and Psychiatry, 34, 551-561.

Frankenhauser, B., and Huxley, A. F. (1964). The action potential in the myelinated nerve fibre of Xenopus laevis as computed on the basis of voltage clamp data. Journal of Physiology, 171, 302-315.

Frisen, L., Hoyt, W. F., Bird, A. L., and Weale, R. A. (1973). Diagnostic uses of the Pulfrich phenomenon. Lancet, 2, 385-386.

Goldman, L., and Albus, J. S. (1968). Computation of the pulse conduction in myelinated fibers: theoretical basis of the velocity-diameter relation. Biophysical Journal, 8, 596-606.

Koles, Z. J., and Rasminsky, M. (1972). A computer simulation of conduction in demyelinated nerve fibres. Journal of Physiology, 227, 351-364.

Rasminsky, M. (1973). The effects of temperature on conduction in demyelinated nerve fibres. Journal of Physio$\log y, 227,323-350$.

Schauf, C. L., and Davis, F. A. (1974). Impulse conduction in multiple sclerosis: a theoretical basis for modification by temperature and pharmacological agents. Journal of Neurology, Neurosurgery, and Psychiatry, 37, 152-161.

Smith, R. S., and Koles, Z. J. (1970). Myelinated nerve fibers: computed effect of myelin thickness on conduction velocity. American Journal of Physiology, 219, 1256-1258.

Waksman, B. H., and Adams, R. D. (1956). A comparative study of experimental allergic neuritis in the rabbit, guinea pig, and mouse. Journal of Neuropathology and Experimental Neurology, 15, 292-333. 